\title{
Elastic scattering of surface states on three-dimensional topological insulators
}

\author{
Jing Wang ${ }^{1,2}$ and Bang-Fen $\mathrm{Zhu}^{1,3, *}$ \\ ${ }^{1}$ State Key Laboratory of Low-Dimensional Quantum Physics, \\ and Department of Physics, Tsinghua University, Beijing 100084, China \\ ${ }^{2}$ Department of Physics, McCullough Building, Stanford University, Stanford, California 94305-4045, USA \\ ${ }^{3}$ Institute of Advanced Study, Tsinghua University, Beijing 100084, China
}

(Dated: November 8, 2018)

\begin{abstract}
Topological insulators as new type of quantum matter materials are characterized by a full insulating gap in the bulk and gapless edge/surface states which are protected by time-reversal symmetry. We propose the interference patterns caused by elastic scattering of defects or impurities are dominated by surface states at the extremal points on the constant energy contour. Within such formalism, we summarize our recent theoretical investigations on elastic scattering of topological surface states by various imperfections, including the non-magnetic impurities, magnetic impurities, step-edges, and various other defects, in comparison with recent related experiments in typical topological materials such as BiSb alloys, $\mathrm{Bi}_{2} \mathrm{Te}_{3}$ and $\mathrm{Bi}_{2} \mathrm{Se}_{3}$ crystals.
\end{abstract}

PACS numbers: 73.20.-r 68.37.Ef 73.43.Cd 72.10.Fk

\section{INTRODUCTION}

The discovery of topological insulators (TIs) has attracted a great deal of attention.12 A threedimensional (3D) TI has a time-reversal invariant band structure with nontrivial topological order, which possesses an energy gap in the bulk and gapless metallic states on the surface. The surface band consist of an odd number of Dirac cones with unconventional spin texture in the surface Brillouin zone. One of the intriguing properties in such spin-textured surface states (SSs) is their insensitivity to spin-independent scattering, which is protected from backscattering by the timereversal symmetry (TRS). Exotic excitations such as Majorana fermions, ${ }^{[3}$ magnetic monopol ${ }^{\sqrt{4}}$ and dynamical axion field $\sqrt{5}$ are predicted to exist as results of topological SSs.

Soon after the theoretical prediction ${ }^{6}, \mathrm{Bi}_{1-x} \mathrm{Sb}_{x}$ alloy with composition $x$ ranged from 0.07 to 0.22 was found to be a TI with five Dirac cones by the angleresolved photoemission spectroscopy (ARPES) ${ }^{7}$ ARPES measurements observed topologically nontrivial SSs, and the spin-resolved ARPES measurements reported the helical spin texture of the massless Dirac fermions in the system .8$]$ However, the SSs in $\mathrm{Bi}_{1-x} \mathrm{Sb}_{x}$ are rather complicated which can hardly be described by a simple model Hamiltonian. Subsequently, a family of binary compounds, $\mathrm{Bi}_{2} \mathrm{Se}_{3}, \mathrm{Bi}_{2} \mathrm{Te}_{3}$ and $\mathrm{Sb}_{2} \mathrm{Te}_{3}$, was discovered and investigated as the second generation of TIs ${ }^{9][12]}$ Among them the $\mathrm{Bi}_{2} \mathrm{Se}_{3}$ contains only a single Dirac cone within the gap as large as $300 \mathrm{meV}$, and as the "hydrogen atom" of TIs is easier to study. The helical spin texture of SSs in $\mathrm{Bi}_{2} \mathrm{Se}_{3}$ is observed to be left-handed. ${ }^{11}$ All these characteristics suggest that direct backscattering between the time-reversal pair of helicity states with opposite momenta and spins is prohibited, and the Anderson localization will not occur in these two-dimensional (2D) helical liquid.

In addition to the ARPES, the low-temperature scan- ning tunneling spectroscopy (STS) as well as microscope (STM) is another important probe in the TI research field, which provides a direct way to study the SSs through measuring the local density of states (LDOS) in the vicinity of various imperfections. Due to the elastic scattering by impurities, the incoming surface wave with a wavevector $\mathbf{k}^{i}$ must be scattered into the outgoing one with $\mathbf{k}^{f}$ on the same constant energy contour (CEC). The quantum interference between the incoming and outgoing waves gives rise to the standing wave oscillation with wavevector $\mathbf{q}=\mathbf{k}^{f}-\mathbf{k}^{i}$, which is often referred as the characteristic scattering wavevector, or the transferred momentum. Energy-resolved Fourier transform STS (FT-STS) can be used to obtain the scattering wavevectors of modulations in the LDOS and detailed information on the symmetry as well as physics of scattering processes for the SS electrons. ${ }^{[13}$ This technique has been proved valuable in studying the SSs on noble metals ${ }^{14}$ and on semiconductor surfaces $\frac{\sqrt{15}}{15}$ and even on the pairing symmetry of the high- $T_{c}$ superconductors 16 and the graphene ${ }^{[17}$ The absence of backscattering is a specular manifestation of the topological nature of the SSs. Different from the edge states in two-dimensional (2D) TIs, in which the forbidden back-scattering means the annihilation of all types of elastic scattering by the spin-independent potentials, the SSs in 3D TIs will experience single-particle elastic scattering associated with all scattering angles except for $180^{\circ}$ backscattering. Thus investigating the quasiparticle interference (QPI) caused by scattering off impurities and defects on the TI surfaces and revealing the topological nature of the SSs through the FT-STS are interesting and useful, in particular based on combination of the theoretical and experimental investigations.

The QPI patterns induced by non-magnetic impurities on surfaces of $\mathrm{Bi}_{x} \mathrm{Sb}_{1-x}{ }^{\frac{18]}{B_{1}}} \mathrm{Bi}_{2} \mathrm{Te}_{3}{ }^{19}$ and $\mathrm{Bi}_{2} \mathrm{Se}_{3} \frac{20}{20}$ together with subsequent theoretical analysis, $\frac{2126}{26}$ did demonstrate the absence of backscattering for the topological SSs. Meanwhile, the LDOS oscillations of SSs 
near step edges on $\mathrm{Bi}_{2} \mathrm{Te}_{3}$ showed a power-law decay with index $-3 / 2,-1 / 2$ and -1 for $\mathrm{SSs}$ at different energy ranges, $26 \mid 27$ while in $\mathrm{Bi}_{2} \mathrm{Se}_{3}$ the decay index is fixed to be $-3 / 2,26 \mid 28$ compared to $-1 / 2$ for the twodimensional electron system (2DES) ${ }^{14}$ The faster decay of QPI pattern is believed to result from the suppression of backscattering in TIs. For magnetic impurities on $\mathrm{Bi}_{2} \mathrm{Te}_{3}$ which breaks the TRS, although the TR violating scattering vectors have been reported, 29 it is still controversial whether the direct backscattering between the time-reversal pair is responsible for the unusual sensitivity to magnetic scattering. 20

So far intensive STM and ARPES investigations have demonstrated the topological properties of SSs; however, a general picture of the QPI on the surface of various TI systems remains elusive, particularly when taking account of the quality of TI samples and the warping effect of the Dirac cone.12 ${ }^{120}$ Here, not only the anisotropic band dispersion of the SSs, but also the warping effect includes coupling between the surface and bulk bands. In this article we will give a comprehensive review on elastic scattering of SSs in various TI systems. The SS-LAphonon quasi-elastic scattering plays an important role in transport of SSs, $\stackrel{31}{b}$ but not affect the QPI pattern significantly, so it will not be reviewed in this article. With the recent experimental results, we present a general formalism to account for the complex QPI on TI surface. We propose that the interference patterns are dominated by SSs at the extremal points on the CEC. In applying this theory to $\mathrm{Bi}_{2} \mathrm{Te}_{3}$ with strong warping effect, we show that, when tuning the bias voltage, for scattering off a non-magnetic impurity the QPI wavevector depends on the shape of Fermi surface sensitively, which varies from conical to $\bar{\Gamma}-\bar{K}$ and finally to $\bar{\Gamma}-\bar{M}$, as the CEC changing from circle to hexagon and finally to snowflake. And the decay index off a step edge also critically depends on the energy of SSs, varying from $-3 / 2$ to $-1 / 2$ and finally to -1 as the energy increases. As for TIs with nearly isotropic Dirac cone, such as $\mathrm{Bi}_{2} \mathrm{Se}_{3}, \frac{32}{32}$ according to our theory the decay index is simply $-3 / 2$. For the scattering off a magnetic impurity, the direct backscattering is allowed due to the TRS breaking. However, we will show such backscattering between time-reversal pairs in the presence of TRS breaking can be hardly seen in LDOS by STM. Therefore, the QPI pattern of magnetic impurity is still similar to that of non-magnetic impurity.

The organization of this paper is as follows. After this introductory section, Sec. III describes the general theory for QPI of 2D SSs. Here we focus on the $\mathrm{Bi}_{2} \mathrm{Se}_{3}$ family for their SSs can be described by a simple Hamiltonian. Section III is devoted to the standing wave due to step edge scattering compared to experiments. Section [V] describes the QPI pattern by scattering off the non-magnetic impurity in combination with related experiments. Section $\mathrm{V}$ describes the scattering of SSs by the magnetic impurity. Section VI summarizes the experimental studies on the scattering off surface fluctuation and one-dimensional buckling. Section VII concludes this paper with a short summary and discussions.

\section{GENERAL THEORY}

The topological $\mathrm{SSs}$ on $\mathrm{Bi}_{2} \mathrm{Te}_{3}$ and $\mathrm{Bi}_{2} \mathrm{Se}_{3}$ have a single Dirac cone near $\bar{\Gamma}$ point in the surface Brillouin Zone on each surface. The effective model describing such topological SSs reads 30

$$
\mathcal{H}_{0}(\mathbf{k})=v\left(\sigma_{x} k_{y}-\sigma_{y} k_{x}\right)+\frac{\lambda}{2}\left(k_{+}^{3}+k_{-}^{3}\right) \sigma_{z},
$$

where $\hbar \equiv 1, k_{ \pm} \equiv k_{y} \pm i k_{x}, v$ is the Dirac velocity, $\lambda$ is the warping parameter, and $\sigma_{i}(i=x, y, z)$ are Pauli matrices acting on spin space. For simplicity, we ignore the particle-hole asymmetry here as it affects the shape of Fermi surface little. The surface band dispersion is

$$
\varepsilon_{ \pm}\left(k_{x}, k_{y}\right)= \pm \sqrt{v^{2} k^{2}+\lambda^{2} k^{6} \sin ^{2}(3 \theta)},
$$

where $\varepsilon_{ \pm}$denotes respectively the upper and the lower energy bands touching at the Dirac point, and $\mathbf{k} \equiv(k, \theta)$ with $\theta$ as the angle between the wave vector $\mathbf{k}$ and $k_{x^{-}}$ axis $(\bar{\Gamma}-\bar{M})$. Defining the characteristic energy $\varepsilon^{*} \equiv$ $v \sqrt{v / \lambda}$ and length $d \equiv \sqrt{\lambda / v}$, in Fig. 1 we plot a set of CEC of the upper band in momentum space with no free parameters for $\mathrm{Bi}_{2} \mathrm{Se}_{3}$ and $\mathrm{Bi}_{2} \mathrm{Te}_{3}$, respectively. For $\mathrm{Bi}_{2} \mathrm{Se}_{3}, \lambda=128 \mathrm{eV} \cdot \AA^{3}$ and $\varepsilon^{*}=0.52 \mathrm{eV},{ }^{9032}$ so that the CEC is nearly isotropic from 0 to $0.42 \varepsilon^{*}(0.22 \mathrm{eV})$. As shown in Fig. 1, when the Fermi energy increases, the shape of CEC evolves from a circle $\left(E=0.31 \varepsilon^{*}\right)$, more hexagon-like $\left(E=0.55 \varepsilon^{*}\right)$, hexagon $\left(E=0.7 \varepsilon^{*}\right)$ and to concave hexagon $\left(E=1.0 \varepsilon^{*}\right)$. In $\mathrm{Bi}_{2} \mathrm{Te}_{3}, \lambda=250 \mathrm{eV} \cdot \AA^{3}$ and $\varepsilon^{*}=0.23 \mathrm{eV}, \frac{12}{, 1}$ so the warping effect is stronger. As shown in Fig. 1, when the Fermi energy increases, the shape of CEC evolves from a hexagon $\left(E=0.7 \varepsilon^{*}\right)$, concave hexagon $\left(E=1.46 \varepsilon^{*}\right)$, more hexagram-like $\left(E=1.91 \varepsilon^{*}\right)$ and to six-pointed shape of snowflake $\left(E=2.4 \varepsilon^{*}\right)$. The evolution of the Fermi surface with respect to energy agrees well with the ARPES results. 12 |32 As we shall see, such changes in the shape of the Fermi surface will have drastic effects on the QPI around an impurity.

We consider the quasiparticle scattering problem within the $T$-matrix approach. For a single non-magnetic or magnetic impurity located at the origin $(\mathbf{r}=0)$ on the surface with the potential

$$
\mathcal{H}_{1}=V \delta(\mathbf{r}) \sigma^{\mu}
$$

where $\sigma^{\mu}=1_{2 \times 2}$ for $\mu=0$ denotes non-magnetic impurity, and $\sigma^{\mu}=\sigma^{x, y, z}$ are the Pauli matrices for $\mu=1,2,3$ denotes magnetic impurity. The LDOS can be expressed as

$$
\begin{aligned}
\rho(\mathbf{r}, \omega) & =-\frac{1}{\pi} \operatorname{Im}[\operatorname{Tr}[G(\mathbf{r}, \mathbf{r}, \omega)]] \\
& =\rho_{0}(\mathbf{r}, \omega)+\delta \rho(\mathbf{r}, \omega)
\end{aligned}
$$




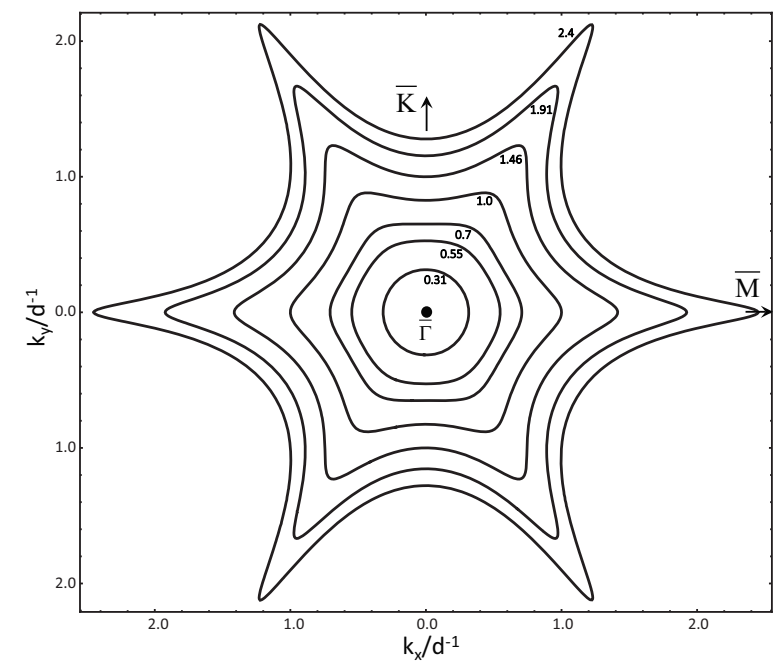

FIG. 1. Unified contours of constant energy and the evolution of Fermi surface of topological SSs in both $\mathrm{Bi}_{2} \mathrm{Se}_{3}$ and $\mathrm{Bi}_{2} \mathrm{Se}_{3}$, where $k_{x}$ and $k_{y}$ axis are in the unit of $\sqrt{v / \lambda}$, and energy is in unit of $\varepsilon^{*}$. The contours with BiSe parameters and $\varepsilon^{*}$ taken from 0.3 to 0.7 are suitable for $\mathrm{Bi}_{2} \mathrm{Se}_{3}$, and those with $\mathrm{Bi}_{2} \mathrm{Te}_{3}$ parameters and $\varepsilon^{*}$ from 0.7 to 1.91 are for BiTe

where $\rho_{0}(\mathbf{r}, \omega)$ is the LDOS of the unperturbed system with $V=0$, and $\delta \rho(\mathbf{r}, \omega)$ is the deviation of the LDOS induced by the perturbation $H_{1}$.

Let the unperturbed surface Green's function be $G_{0}$, the perturbed Green's function can be expressed as

$$
G\left(\mathbf{r}, \mathbf{r}^{\prime}, \omega\right)=G_{0}\left(\mathbf{r}-\mathbf{r}^{\prime}, \omega\right)+G_{0}(\mathbf{r}, \omega) T(\omega) G_{0}\left(-\mathbf{r}^{\prime}, \omega\right),
$$

with

$$
\begin{aligned}
G_{0}(\mathbf{r}, \omega) & =\int^{\Lambda} \frac{d^{2} k}{(2 \pi)^{2}} e^{i \mathbf{k} \cdot \mathbf{r}} G_{0}(\mathbf{k}, \omega), \\
G_{0}(\mathbf{k}, \omega) & =\left[\omega+i \eta-\mathcal{H}_{0}(\mathbf{k})\right]^{-1},
\end{aligned}
$$

where $\Lambda$ is the energy cutoff when integrating over momentum, and $T$ is given by

$$
T(\omega)=V \sigma^{\mu}\left[1-G_{0}(\omega) V \sigma^{\mu}\right]^{-1} .
$$

Here we assume the translation symmetry in the unperturbed system and the momentum independent scattering potential. The spatial modulation of the LDOS is induced by $\mathcal{H}_{1}$ in the vicinity of impurities. To resolve the wave vector of the modulation of interference, it is convenient to calculate the Fourier-transformed LDOS

$$
\delta \rho(\mathbf{q}, \omega)=\int d^{2} r e^{-i \mathbf{q} \cdot \mathbf{r}} \delta \rho(\mathbf{r}, \omega) .
$$

From the real-space LDOS, one can get the power-law decay as a function of distance away from the impurity center $\delta \rho(\mathbf{r}, \omega) \propto 1 / r^{\alpha}$. While from the $q$-space LDOS, one can get the allowed wave vector $\mathbf{q}$ and the relative intensities for various scattering processes experienced by the SS electrons. Both the real-space and $q$-space information can be obtained by STM.

For the case of scattering by a step edge, the scattering potential is taken as $V \delta(x)$. It is straightforward to compute the LDOS by the standard procedure described above. However, without loss of generality, it is more convenient to treat the scattering problem by using an analogy of the elementary scattering problem with a barrier potential, either in 1D (step-edge) or 2D with rotational symmetry (magnetic or non-magnetic impurity), which is directly based on the wave function point of view. We will discuss the formalism in Sec. III A and Sec. IVA. respectively.

\section{SCATTERING BY STEP-EDGE}

\section{A. Theory}

Let us first consider the scattering of SSs by a step edge. The step on a surface along a crystallographic axis in principle reduces the interference problem to a $1 \mathrm{D}$ phenomenon. The step edge in a 2D conventional Fermi gas is known to give rise to Friedel oscillation at a fixed energy in the LDOS ${ }^{14}$ Since the step edge scattering is a type of elastic scattering, the incoming SS with wave vector $\mathbf{k}^{i}$ and the outgoing one with $\mathbf{k}^{f}$ are on the same CEC. Assuming the step edge along the $y$-direction, the $k_{y}$ component of the wave vectors should be conserved in the scattering process, i.e. $k_{y}^{i}=k_{y}^{f} \equiv k_{y}$. The interference between the incoming and outgoing waves gives rise to the standing wave oscillation in the $x$-direction. The total LDOS is the sum of contributions from all these oscillations from the SSs on a CEC. For a given energy $E$, we can integrate over $k_{y}$ on the entire CEC and express the LDOS explicitly as

$$
\delta \rho(E, x)=\operatorname{Re}\left[\oint_{E} \frac{2 r_{c}}{1+\left|r_{c}\right|^{2}} \xi_{i}^{\dagger} \xi_{f} e^{i\left(k_{x}^{f}-k_{x}^{i}\right) x} d k_{y}\right],
$$

where $r_{c}$ is the reflection coefficient of the potential barrier of the step-edge, and $\xi$ denotes the spin wave function of the SS of the form $\xi e^{i k_{x} x+i k_{y} y}$.

A pair of states $\mathbf{k}^{i}$ and $\mathbf{k}^{f}$ scattered by the step along $y$-direction results in a standing wave with spatial period of $2 \pi /\left(k_{x}^{f}-k_{x}^{i}\right) \equiv 2 \pi / \Delta k_{x}$. Since the oscillation period is different for different value of $k_{y}$, a large number of scattering pairs will interfere destructively, and only the pair whose period is stationary with respect to small variation in $k_{y}$ may make dominant contribution to the LDOS oscillations. Such pair of points $\left[\left(k_{x 0}^{i}, k_{y 0}\right)\right.$ and $\left.\left(k_{x 0}^{f}, k_{y 0}\right)\right]$ on CEC are called the extremal points (EPs) ${ }^{33}$ Namely, $\partial \Delta k_{x} /\left.\partial k_{y}\right|_{k_{y 0}}=0$. And $\Delta k_{x 0}$ is the characteristic scattering wavevector which critically depends on the geometry of CEC. Other standing waves will cancel at large $x$ because of mutual interference. The spatial dependence of LDOS oscillations in Eq. (10) can be evaluated by expanding the relevant quantities around each pair of EPs. 


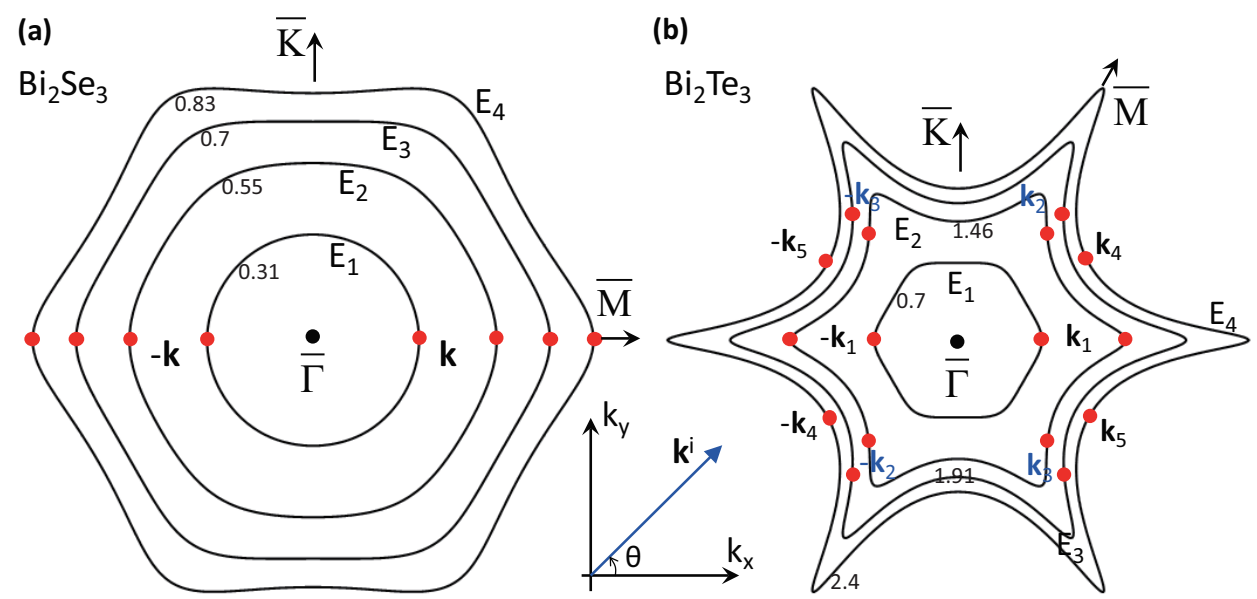

FIG. 2. (color online) Illustration of scattering off the step edge along $y$-direction. The LDOS oscillation is dominated by scattering between the EP pairs (red dots) on CEC. (a) The shape of CEC in $\mathrm{Bi}_{2} \mathrm{Se}_{3}$ evolves from circle, hexagon to concave hexagon as energy increases. It has a single pair of EPs at $(\mathbf{k},-\mathbf{k})$ and only one type of oscillation pattern appears with different bias voltages. (b) In $\mathrm{Bi}_{2} \mathrm{Te}_{3}$, as energy increases, CEC evolves from hexagon, concave hexagon to concave hexagram, corresponding to the pair of EPs, first at $\left(\mathbf{k}_{1},-\mathbf{k}_{1}\right)$; then at $\left(\mathbf{k}_{1},-\mathbf{k}_{1}\right),\left(\mathbf{k}_{2},-\mathbf{k}_{3}\right),\left(\mathbf{k}_{3},-\mathbf{k}_{2}\right)$; then at $\left(\mathbf{k}_{2},-\mathbf{k}_{3}\right),\left(\mathbf{k}_{3},-\mathbf{k}_{2}\right)$; finally at $\left(\mathbf{k}_{4},-\mathbf{k}_{5}\right)$ and $\left(\mathbf{k}_{5},-\mathbf{k}_{4}\right)$. Different types of oscillation pattern appear at different bias voltages.

Let $k_{y}=k_{y 0}+\delta k_{y}$, then $\Delta k_{x}=\Delta k_{x 0}+\sum_{n} \Delta k_{x n} \delta k_{y}^{n}$, $r=\sum_{l} \eta_{l} \delta k_{y}^{l}$, and $\xi_{i}^{\dagger} \xi_{f}=\sum_{m} \chi_{m} \delta k_{y}^{m}$. To the leading order of $\delta k_{y}$, the LDOS varies at long distance as

$$
\begin{aligned}
\delta \rho(E, x) & \simeq \operatorname{Re}\left[\sum_{\mathrm{EPs}} \int_{E} 2 r /\left(1+|r|^{2}\right) \xi_{i}^{\dagger} \xi_{f} e^{i\left(k_{x}^{f}-k_{x}^{i}\right) x} d k_{y}\right], \\
& \sim \sum_{\mathrm{EPs}}\left|g \eta_{a} \chi_{b} c\right| \cos \left(\Delta k_{x 0} x+\phi_{s}\right) x^{-\frac{a+b+1}{c}},
\end{aligned}
$$

where $\quad a=\min (l), \quad b=\min (m), \quad c=\min (n), \quad g \quad=$ $\oint_{E} d k^{\prime} k^{(a+b-c+1) / c} e^{i \Delta k_{x c} k^{\prime}}$, and $\phi_{s}$ is the initial phase of each pair of EPs. The decay behavior of LDOS in Eq. (11) is valid as long as $x \gg \Delta k_{x 0}^{-1}$. The decay index associated with a pair of EPs is given by $(a+b+1) / c$, which is solely determined by the properties of the scattering wave function around the EPs on a given CEC.

The step edge is always along the close packed $\bar{\Gamma}-\bar{K}$ direction. As shown in Fig. 2(a), In $\mathrm{Bi}_{2} \mathrm{Se}_{3}$, when the Fermi energy increases, the shape of CEC evolves from a circle $\left(E_{1}=0.31 \varepsilon^{*}\right)$, more hexagon-like $\left(E_{2}=0.55 \varepsilon^{*}\right)$, hexagon $\left(E_{3}=0.7 \varepsilon^{*}\right)$ and to concave hexagon $\left(E_{4}=\right.$ $\left.0.83 \varepsilon^{*}\right)$. In a wide range of energy only a single pair of EPs exists at $(\mathbf{k},-\mathbf{k})$, so the characteristic wave vector is always equal to $2 \mathbf{k}$ and $c=2$. In $\mathrm{Bi}_{2} \mathrm{Te}_{3}$, the warping effect is stronger, and the power law decay behavior is more complicated. As shown in Fig. 2(b), EPs evolve with the energy as follows: Single pair of $\operatorname{EPs}\left(\mathbf{k}_{1},-\mathbf{k}_{1}\right)$ at $E_{1}=0.7 \varepsilon^{*}$; Multiple pairs of EPs $\left(\mathbf{k}_{1},-\mathbf{k}_{1}\right),\left(\mathbf{k}_{2},-\mathbf{k}_{3}\right)$ and $\left(\mathbf{k}_{3},-\mathbf{k}_{2}\right)$ at $E_{2}=1.46 \varepsilon^{*}>E_{c} \equiv 3^{1 / 3} \sqrt{11 / 9} \varepsilon^{*} \simeq$ $1.45 \varepsilon^{*}$; Two pairs of EPs $\left(\mathbf{k}_{2},-\mathbf{k}_{3}\right)$ and $\left(\mathbf{k}_{3},-\mathbf{k}_{2}\right)$ survive at $E_{3}=1.91 \varepsilon^{*}$, as the SSs along the $\bar{\Gamma}-\bar{M}$ direction merge into the bulk conduction band; No EPs at all at $E_{4}=$
$2.4 \varepsilon^{*}$, because the SSs separate from bulk states only in the very vicinity along $\bar{\Gamma}-\bar{K}$ direction on the Fermi surface as observed in the ARPES experiment ${ }^{[12}$ In this case, scattering between SSs around $\left(\mathbf{k}_{4},-\mathbf{k}_{5}\right)$ and $\left(\mathbf{k}_{5},-\mathbf{k}_{4}\right)$ will mostly contribute to LDOS oscillations, but they are not EPs and $c=1$. Thus in $\mathrm{Bi}_{2} \mathrm{Te}_{3}$ the characteristic wave vector and the LDOS oscillation period critically depend on the energy of SSs via varying the bias in the STS. In most cases we have parameter $c=2$ except for Fermi energy as high as $E_{4}(c=1)$.

For the incoming state with wave vector $\mathbf{k}^{i}=\left(k^{i}, \theta^{i}\right)$ and energy $\varepsilon_{+}\left(\mathbf{k}^{i}\right)$, the inner product of two spin wave functions $\xi_{i}^{\dagger} \xi_{f}=\sin \theta^{i}+i \lambda\left(k^{i}\right)^{3} \sin \left(3 \theta^{i}\right) \cos \theta^{i} / \varepsilon_{+}\left(\mathbf{k}^{i}\right)$. It vanishes only for EPs associated with zero $\theta$, when the spins are exactly anti-parallel for the time-reversal pairs of $(\mathbf{k},-\mathbf{k})$ and $\left(\mathbf{k}_{1},-\mathbf{k}_{1}\right)$. Since $b$ is the lowest power for $\xi_{i}^{\dagger} \xi_{f}$, for the time-reversal pairs $b \geq 1$; otherwise $b=0$. Thus, $b=1$ in $\mathrm{Bi}_{2} \mathrm{Se}_{3}$; while in $\mathrm{Bi}_{2} \mathrm{Te}_{3}, b=1$ for the pair $\left(\mathbf{k}_{1},-\mathbf{k}_{1}\right)$, and $b=0$ for other pairs of EPs.

Assuming the step edge potential is $V(x)=0$ for $x<0$ and $V(x)=-V_{0}\left(V_{0}>0\right)$ for $x>0$, by matching the boundary condition at the edge the reflection coefficient can be obtained as

$$
r\left(\theta^{i}\right)=(-i) \frac{e^{-i\left(\theta^{i}-\theta^{t}\right) / 2}-\beta\left(\theta^{i}\right) e^{i\left(\theta^{i}-\theta^{t}\right) / 2}}{e^{i\left(\theta^{i}+\theta^{t}\right) / 2}+\beta\left(\theta^{i}\right) e^{-i\left(\theta^{i}+\theta^{t}\right) / 2}},
$$

where $\left(k^{t}, \theta^{t}\right)$ is the momentum of the transmitted state,

$$
\beta\left(\theta^{i}\right)=\frac{\varepsilon_{+}\left(\mathbf{k}^{i}\right) / k^{i}+\lambda\left(k^{i}\right)^{2} \sin \left(3 \theta^{i}\right)}{\varepsilon_{+}\left(\mathbf{k}^{t}\right) / k^{t}+\lambda\left(k^{t}\right)^{2} \sin \left(3 \theta^{t}\right)},
$$

$\varepsilon_{+}\left(\mathbf{k}^{i}\right)=\varepsilon_{+}\left(\mathbf{k}^{t}\right)-V_{0}$, and $\theta^{t}\left(\theta^{i}\right)=-\theta^{t}\left(-\theta^{i}\right)$. Due to the constraint by TRS, $r\left(\theta^{i}\right)=-r\left(-\theta^{i}\right)$, and the normal 
reflection $r\left(\theta^{i}=0\right)=0$, which means the absence of backscattering. $a$ is the lowest power for $r$, thus $a=1$ for the backscattering pair of $(\mathbf{k},-\mathbf{k})$ in $\mathrm{Bi}_{2} \mathrm{Se}_{3}$ and $\left(\mathbf{k}_{1},-\mathbf{k}_{1}\right)$ in $\mathrm{Bi}_{2} \mathrm{Te}_{3}$, and $a=0$ for other pairs in $\mathrm{Bi}_{2} \mathrm{Te}_{3}$.

In short, the algebraical decay index is $3 / 2$ for $(\mathbf{k},-\mathbf{k})$ and $\left(\mathbf{k}_{1},-\mathbf{k}_{1}\right)$ pairs, $1 / 2$ for $\left(\mathbf{k}_{2},-\mathbf{k}_{3}\right)$ and $\left(\mathbf{k}_{3},-\mathbf{k}_{2}\right)$ pairs, and 1 for $\left(\mathbf{k}_{4},-\mathbf{k}_{5}\right)$ and $\left(\mathbf{k}_{5},-\mathbf{k}_{6}\right)$ pairs. Therefore, the LDOS oscillations of the $\mathrm{SSs}$ in $\mathrm{Bi}_{2} \mathrm{Se}_{3}$ should decay as $x^{-3 / 2}$ in a wide range of energy (as long as $E<0.76 \mathrm{eV}$ ), much faster than $x^{-1 / 2}$ in 2DES ${ }^{14} \mathrm{On} \mathrm{Bi}_{2} \mathrm{Te}_{3}$ surfaces, as the bias increases, LDOS oscillations decay first as $x^{-3 / 2}(E<0.33 \mathrm{eV})$, then as a combination of $x^{-3 / 2}$ and $x^{-1 / 2}$, then as $x^{-1 / 2}$, and finally $x^{-1}$.

\begin{tabular}{cc}
\hline \hline & decay power \\
\hline $0<E<1.45 \varepsilon^{*}$ & $x^{-3 / 2}$ \\
$1.45 \varepsilon^{*}<E<\varepsilon_{1}$ & $x^{-3 / 2}, x^{-1 / 2}$ \\
$\varepsilon_{1}<E<\varepsilon_{2}$ & $x^{-1 / 2}$ \\
$\varepsilon_{2}<E<\varepsilon_{\max }$ & $x^{-1}$ \\
\hline \hline
\end{tabular}

TABLE I. Index of power law decay of standing waves of SSs scattering by step-edges. $\varepsilon_{1}$ denotes the energy at which the SSs along $\bar{\Gamma}-\bar{M}$ begin to merge into bulk conduction band, $\varepsilon_{2}$ denotes the energy where the SSs only exist in the very vicinity along $\bar{\Gamma}-\bar{K} . \quad \varepsilon_{\max }$ is the energy upper bound for the surface Dirac electron.

\section{B. Experiments}

Kapitulnik's group performed STM and STS study on Sn- and Cd-doped $\mathrm{Bi}_{2} \mathrm{Te}_{3}$ crystals. ${ }^{27}$ By analyzing the oscillation of LDOS near a step-edge, they showed that topological SS oscillations are strongly damped compared to conventional SSs. This is another hallmark of the severe suppression of backscattering, hence supporting the TRS protected $\mathrm{SSs}$ in $\mathrm{Bi}_{2} \mathrm{Te}_{3}$. An interesting observation in experiments is the emergence of pronounced oscillations with a distinct wave vector at higher energies, which may result from a hexagonal warping of the surface band structure.

Xue's group reported the first interference fringes at the step edges on $\mathrm{Bi}_{2} \mathrm{Se}_{3}$ surface thanks to their high quality sample $\frac{1926}{26}$ By analyzing decay power of standing wave oscillations across a step on $\mathrm{Bi}_{2} \mathrm{Se}_{3}$, they showed that the oscillations decay index is $x^{-3 / 2}$, much faster than in 2D metal $x^{-1 / 214}$ This confirms the suppression of backscattering of topological SSs in $\mathrm{Bi}_{2} \mathrm{Se}_{3}$ due to TRS. Such faster decay power of $-3 / 2$ in $\mathrm{Bi}_{2} \mathrm{Se}_{3}$ is

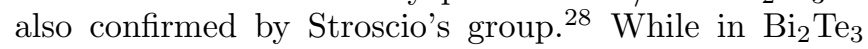
with strongly warped surface band, they clearly reveal the variation of the QPI decay index with the bias voltage. As shown in Table 1, as the energy of SS increases, the power index varies from $x^{-3 / 2}$ to a mixture of $x^{-3 / 2}$ and $x^{-1 / 2}$, to $x^{-1 / 2}$, and finally to $x^{-1}$. The $x^{-1 / 2}$ decay indicates that the suppression of backscattering due to TRS does not necessarily lead to a spatial decay rate faster than that in the normal 2DES. As shown in Ref.26, the agreement between experiment of Xue's group and our theoretical prediction is surprisingly perfect.

\section{NON-MAGNETIC IMPURITY}

\section{A. Theory}

Now we switch from the $1 \mathrm{D}$ scattering to $2 \mathrm{D}$ scattering. We first consider the interference patterns of topological SSs due to a non-magnetic impurity. The interference wave vectors of the QPI pattern can be simply understood by exploring the standing wave formation mechanism. Suppose an incident wave $\psi_{i} \propto e^{i \mathbf{k}^{i} \cdot \mathbf{r}} / \sqrt{r}$ is scattered into $\psi_{f} \propto f(\theta) e^{i \mathbf{k}^{f} \cdot \mathbf{r}} / \sqrt{r}$ by the impurity potential. The resultant LDOS oscillation is

$$
\delta \rho(\mathbf{r}, \omega) \propto \sum_{\mathbf{k}^{i}, \mathbf{k}^{f}} \frac{1}{r} f(\theta) e^{i\left(\mathbf{k}^{f}-\mathbf{k}^{i}\right) \cdot \mathbf{r}},
$$

with $f(\theta)$ denoting the scattering amplitude and $r$ denoting the distance away from the impurity center. Also because of the elastic scattering, $\mathbf{k}^{i}$ and $\mathbf{k}^{f}$ are on the same CEC. There are various scattering directions between incoming and outgoing SSs, but most of these processes interfere destructively, contributing little to the standing waves. The interference pattern are dominated by the pair of EPs on the CEC either, 26 which resembles the Fermat's principle in optics. The characteristic scattering wavevector of the QPI pattern at large distance in a given direction $\hat{r}$ comes from scattering between states at EPs on the Fermi surface, where the Fermi velocity is parallel to $\hat{r}$.

For the circle-like CEC shown in Fig. 3(a), the EP pair only exists at $\mathbf{k}$ and its time-reversal partner $\mathbf{- k}$. There are many such pairs, for example, $\left(\mathbf{k}_{1},-\mathbf{k}_{1}\right),\left(\mathbf{k}_{2},-\mathbf{k}_{2}\right)$, and $\left(\mathbf{k}_{3},-\mathbf{k}_{3}\right)$, etc. Let $\vec{q}_{1}$ represent the backscattering vector between $\mathbf{k}$ and $-\mathbf{k}$. Obviously, there is no dominating direction of $\vec{q}_{1}$ in the circle case. The two states $|\mathbf{k}, \uparrow\rangle$ and $|-\mathbf{k}, \downarrow\rangle$, which are related by the TRS as $|-\mathbf{k}, \downarrow\rangle=\mathcal{T}|\mathbf{k}, \uparrow\rangle$, carry opposite spins, hence

$$
\begin{aligned}
\langle-\mathbf{k}, \downarrow|\hat{U}| \mathbf{k}, \uparrow\rangle & =-\langle\mathbf{k}, \uparrow|\hat{U}|-\mathbf{k}, \downarrow\rangle^{*} \\
& =-\langle-\mathbf{k}, \downarrow|\hat{U}| \mathbf{k}, \uparrow\rangle=0 .
\end{aligned}
$$

Thus the $q_{1}$-scattering between two Krames degenerate states is forbidden. Here $\mathcal{T}$ is the time-reversal operator with $\mathcal{T}^{2}=-1$, and $\hat{U}$ is a TR-independent operator representing the non-magnetic scattering potential. Therefore, the LDOS oscillation vanishes to leading order. Taking the next order into account, it decays algebraically as $1 / r^{2}$. For a convex CEC shown in Fig. 3(b), there exist only two kinds of characteristic wave vectors: $\vec{q}_{1}$ along $\bar{\Gamma}-\bar{K}$ and $\vec{q}_{2}$ along $\bar{\Gamma}-\bar{M}$ direction. Both of them 
(a)

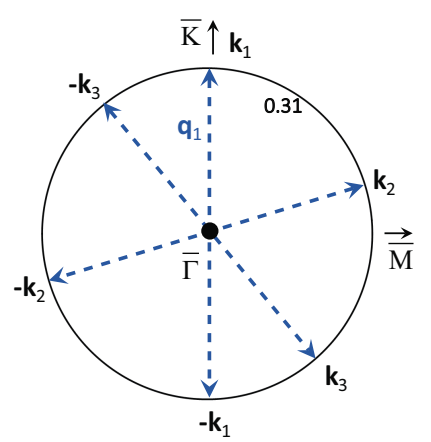

(d)

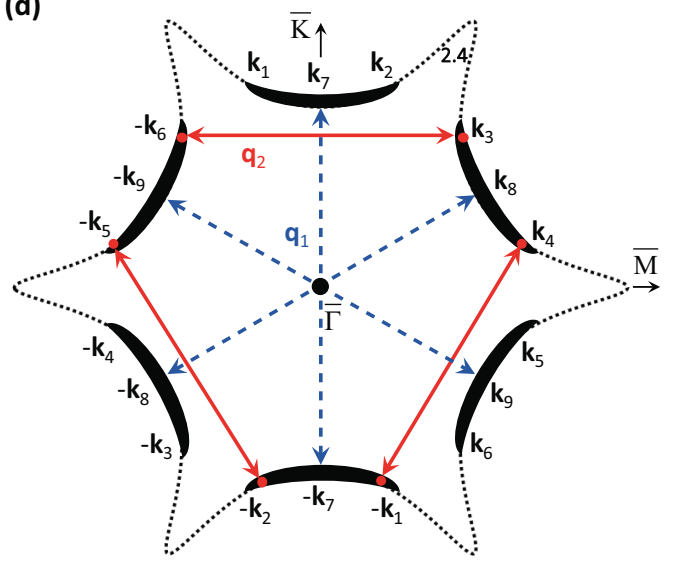

(b)

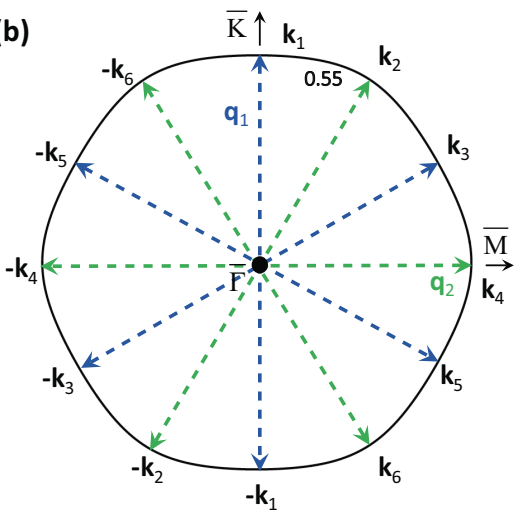

(c)

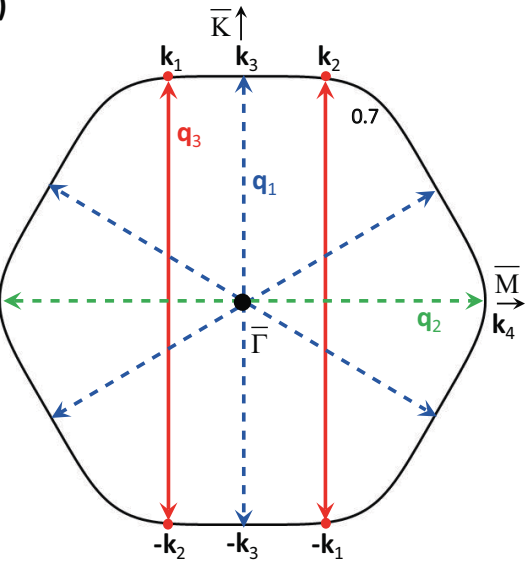

FIG. 3. (color online) Illustration of scattering geometry due to a non-magnetic impurity. The LDOS oscillation is dominated by scattering between the pair of EPs on CEC. (a) For the CEC of circle, the pair of EPs is the SS with $\mathbf{k}$ and its time-reversal state $-\mathbf{k}$, such as $\left(\mathbf{k}_{1},-\mathbf{k}_{1}\right),\left(\mathbf{k}_{2},-\mathbf{k}_{2}\right)$ and $\left(\mathbf{k}_{3},-\mathbf{k}_{3}\right)$ etc. The QPI pattern in the $q$-space is circular. (b) When the CEC evolves into convex, two types of forbidden characteristic wave vectors, $\vec{q}_{1}$ (dashed blue) and $\vec{q}_{2}$ (dashed green), connect the time-reversal pairs. (c) For the CEC of hexagon, $\vec{q}_{1}$ and $\vec{q}_{2}$ are forbidden, while $\vec{q}_{3}$ (solid red), in the direction of $\bar{\Gamma}-\bar{K}$, dominates the QPI pattern. (d) For the hexagram CEC, the regions with high DOS are denoted by bold lines along the direction of $\bar{\Gamma}-\bar{K}$. Two types of characteristic scattering wave vectors include the forbidden $\vec{q}_{1}$ (dashed blue) and the allowed $\vec{q}_{2}$ (solid red) which connects a pair of EPs. Thus different oscillation wave vectors appear in the FT-STS with different bias voltages.

connect a pair of TRS states whose scattering is prohibited, thus the LDOS oscillation decays as $1 / r^{2}$. For a hexagon-like CEC shown in Fig. 3(c), there exist three kinds of characteristic wave vectors: $\vec{q}_{1}, \vec{q}_{3}$ along $\bar{\Gamma}-\bar{K}$ and $\vec{q}_{2}$ along $\bar{\Gamma}-\bar{M}$. Obviously, the $\vec{q}_{1}$ and $\vec{q}_{2}$ scattering are forbidden for they connect pairs of TRS states. The $\overrightarrow{q_{3}}$ connects a pair of states at EP, which dominates the QPI, and it decays as $1 / r$. With the concave hexagram Fermi surface as shown in Fig. 3(d), there exist only two kinds of characteristic wave vectors: $\vec{q}_{1}$ along $\bar{\Gamma}-\bar{K}$ and $\vec{q}_{2}$ along $\bar{\Gamma}-\bar{M}$ direction. $\vec{q}_{1}$ is forbidden, and $\vec{q}_{2}$ dominates the QPI with decay index $1 / r$. The characteristic wave vector and decay power of the QPI pattern is summarized in Table 2.

The scattering theory of the EPs can better explain the experiments. In the scattering configuration for $\mathrm{Bi}_{2} \mathrm{Te}_{3}$ as shown in Fig. 3(d), by numerical calculations we obtain the allowed scattering vector $q_{2}$, which varies lin-

\begin{tabular}{ccc}
\hline \hline & wave vector & decay power \\
\hline $0<E<0.4 \varepsilon^{*}$ & - & $1 / r^{2}$ \\
$0.4 \varepsilon^{*}<E<0.69 \varepsilon^{*}$ & $\bar{\Gamma}-\bar{K}, \bar{\Gamma}-\bar{M}$ & $1 / r^{2}$ \\
$0.69 \varepsilon^{*}<E<1.45 \varepsilon^{*}$ & $\bar{\Gamma}-\bar{K}$ & $1 / r$ \\
$1.45 \varepsilon^{*}<E<\varepsilon_{\max }$ & $\bar{\Gamma}-\bar{M}$ & $1 / r$ \\
\hline \hline
\end{tabular}

TABLE II. Characteristic wave vector and power laws of QPI pattern for a non-magnetic impurity. $\varepsilon_{\max }$ is the energy upper bound for the surface Dirac electron.

early with the energy as $q_{2}=1.5 \bar{k}$ (rather than $\sqrt{3} \bar{k}^{19}$ ), where $\bar{k}$ is the length of $\bar{\Gamma}-\mathbf{k}_{7}$. Together with the STM data, we deduce the Dirac velocity along $\bar{\Gamma}-\bar{K}$ should be $v=4.15 \times 10^{5} \mathrm{~m} / \mathrm{s}$ (instead of $4.8 \times 10^{5} \mathrm{~m} / 19$ ), in 
agreement with ARPES result $v=4.05 \times 10^{5} \mathrm{~m} / \mathrm{s} ! 12$. In $\mathrm{Bi}_{2} \mathrm{Se}_{3}$, the CEC is circle-like up to $0.22 \mathrm{eV}$ and the characteristic scattering wave vector is always along the diameter of the circle. Thus, we expect the Fourier transformation of $\mathrm{LDOS}$ on $\mathrm{Bi}_{2} \mathrm{Se}_{3}$ surface is ring-like.

\section{B. Experiments}

Yazdani's group reported their STM study of disorder scattering of $\mathrm{SSs}$ on $\mathrm{Bi}_{1-x} \mathrm{Sb}_{x}$ alloy ${ }^{18}$ They used the energy-resolved FT-STS to draw the interference wave vectors in $q$-space. By comparing with spin-resolved ARPES data, a comprehensive analysis of QPI patterns indicated there were eight allowed scattering wave vectors, excluding those connecting states of opposite spins. With the spin-selective scattering they explicitly proved the TRS of topological SSs in this material. More recently, STM experiments further demonstrated that the topological SSs could penetrate barriers while maintaining their extended nature in $\mathrm{Sb} 34$

Xue's group reported the first direct STM imaging of standing waves on the $\mathrm{Bi}_{2} \mathrm{Te}_{3}$ (111) surface ${ }^{19}$ The interference fringes are caused by scattering off non-magnetic $\mathrm{Ag}$ atoms of the topological states. The Dirac dispersion relation of $E(k)$ of SSs is confirmed by the voltage dependent QPI patterns. The experiment of Xue's group indicates the backscattering of SSs is completely suppressed, which is a direct proof of the topological nature of the $\mathrm{SSs}$ in $\mathrm{Bi}_{2} \mathrm{Te}_{3}$. It worths mentioning that the allowed interference wave vectors in $\mathrm{Bi}_{2} \mathrm{Te}_{3}$ come from the intravalley scattering, i.e. the SSs are within the single Dirac cone; while in $\mathrm{Bi}_{1-x} \mathrm{Sb}_{x}$ alloy the scattering wave vectors are related to the intervalley scattering between different Dirac cones.

Later, Yazdani's group reported the STM study of the QPI patterns on the surface of Ca-doped $\mathrm{Bi}_{2} \mathrm{Te}_{3}$ at several bias voltages. ${ }^{[2]}$ The standing wave patterns in $q$ space exhibit six strong peaks along the $\bar{\Gamma}-\bar{M}$ direction for SSs with high energies, and circular patterns with lower ones. This can be well understood on the basis of the Fermi surface shape and its associated spin texture. The warped Fermi surface in $\mathrm{Bi}_{2} \mathrm{Te}_{3}$ dominates scattering of SSs with high energies relative to the Dirac point. In the low-energy region where the dispersion of SSs is conic and the scattering results in a simple circular pattern in the Fourier-transformed QPI. The low energy scattering pattern is also consistent with the helical spin texture of the topological SSs that still allows all scattering processes except for the direct backscattering.

\section{CLASSICAL MAGNETIC IMPURITY}

\section{A. Theory}

The TRS in TIs is broken by doped magnetic impurities, so that in principle the backscattering process will occur, which is prohibited in TI with non-magnetic impurities only. Here we focus on the effects of a classical magnetic impurity, which implies the Kondo physics can be ignored.

Suppose the scattering potential of an magnetic impurity is

$$
\mathcal{H}_{1}=V_{i} \sigma^{i} \delta(\mathbf{r})=J_{i}^{\mathrm{eff}}\left\langle S_{i}\right\rangle \sigma^{i} \delta(\mathbf{r}),
$$

where $\sigma^{i}(i=x, y, z)$ are the Pauli matrices denoting the electron spin, $\left\langle S_{i}\right\rangle$ is the expectation value of the local magnetic moment, and $J_{i}^{\text {eff }}$ denotes the effective exchange coupling between electron spin and local magnetic moment. As indicated by Eq. 15, with TRS, the backscattering is absent between a pair of TR-states of $|\mathbf{k}, \uparrow\rangle$ and $|-\mathbf{k}, \downarrow\rangle$. It is thus straightforward to prove the TRS-breaking potential such as the potential of magnetic impurities can remove such a constraint on backscattering, because

$$
\mathcal{T} \sigma_{i} \mathcal{T}^{-1}=-\sigma_{i}
$$

and

$$
\langle-\mathbf{k}, \downarrow|\hat{U}| \mathbf{k}, \uparrow\rangle \neq 0 .
$$

However, such backscattering between TR pairs can hardly be observed in the LDOS spectra.? Instead of having $\delta \rho_{\uparrow}(\mathbf{q}, \omega)=\delta \rho_{\downarrow}(\mathbf{q}, \omega)$ in the non-magnetic impurity case, here we have $\delta \rho_{\uparrow}(\mathbf{q}, \omega) \approx-\delta \rho_{\downarrow}(\mathbf{q}, \omega)$. Suppose the magnetic moment of the impurity is along the $z$ direction, then the spin-up electrons and spin-down electrons experience opposite scattering potentials. Thus to the lowest order of perturbation theory, the scattering amplitudes of the spin-up and spin-down electrons have opposite sign, so that the total interference pattern of the charge density vanishes almost everywhere.

$$
\begin{aligned}
\delta \rho^{1}(\mathbf{q}, \omega) & \approx \int \frac{d^{2} k}{4 \pi} \operatorname{Tr}\left[G_{0}(\mathbf{k}, \omega) \mathcal{H}_{1} G_{0}(\mathbf{k}+\mathbf{q}, \omega)\right] \\
& =\sum_{i} V_{i} \int \frac{d^{2} k}{4 \pi} \operatorname{Tr}\left[G_{0}(\mathbf{k}, \omega) \sigma_{i} G_{0}(\mathbf{k}+\mathbf{q}, \omega)\right] \\
& =0
\end{aligned}
$$

This argument no longer holds if higher orders of perturbation are considered. The higher order perturbations to the scattering amplitude would lead to similar results as in non-magnetic impurities.

\section{B. Experiments}

Recently, Madhavan's group reported the first TR violating scattering vectors in magnetically doped TI of $\mathrm{Bi}_{2} \mathrm{Fe}_{2-x} \mathrm{Te}_{3}$ by using FT-STM dI/dV maps. ${ }^{29}$ Similar to previous studies $\frac{19}{19}$ a sixfold symmetric pattern with intensity centered along the $\bar{\Gamma}-\bar{M}$ directions are observed above $150 \mathrm{meV}$. Remarkably, at low energies starting around $60 \mathrm{meV}$, a new set of scattering vectors centered 
along $\bar{\Gamma}-\bar{K}$ directions have appears, not reported in prior studies. By comparing with ARPES results, they claimed that the new scattering channels arose from the forbidden backscattering. However, at the energy $140 \mathrm{meV}$ where scattering vector along $\bar{\Gamma}-\bar{K}$ emerges for the CEC similar to the hexagon in Fig. 3(c). The scattering vector by a non-magnetic or magnetic impurity should be along the $\bar{\Gamma}-\bar{K}$ direction solely due to the shape of Fermi surface. Therefore, whether these new scattering channels arise from the forbidden backscattering or not is still unsettled.

Later, Yazdani's group reported the standing wave patterns on Mn-doped $\mathrm{Bi}_{2} \mathrm{Te}_{3}$ and $\mathrm{Bi}_{2} \mathrm{Se}_{3}$. 20 The detailed Fourier analysis of the QPI patterns in $q$-space shows that the short wavelength scattering in different samples have similar results, which is independent of whether the scattering dopant is magnetic or not, or even if magnetic order is established. The FT-QPI pattern consists of six strong peaks along the $\bar{\Gamma}-\bar{M}$ directions at high energies and circular patterns at lower ones. These results strongly support that in the presence of weak magnetic impurities the backscattering between time-reversal pairs can hardly be seen in the LDOS.

Kimura's group reported the QPI pattern induced by cobalt adatoms on the $\mathrm{Bi}_{2} \mathrm{Se}_{3}$ surface by STM ${ }^{35}$ They find that Co atoms are selectively adsorbed on top of Se sites and act as strong scatter centers on the surface, generating anisotropic standing waves along $\bar{\Gamma}-\bar{M}$ directions. However, the long-range ferromagnetic order is found to be absent, and the Dirac cone of SSs remains gapless. The anisotropy of the standing waves at high bias voltage is ascribed to the heavily warped CEC of unoccupied states. At low voltage the QPI patterns due to the occupied states near Dirac cone vanishes, which suggests that the time-reversal protection of the topological SSs persists even in the presence of Co impurities.

\section{OTHER DEFECTS}

Yandani's group studied the influence of the bulkoriginated disorder potential on the short-wavelength interference patterns caused by the surface impurities in
$\mathrm{Bi}_{1.95} \mathrm{Mn}_{0.05} \mathrm{Te}_{3}{ }^{20}$ They find the wave vector shift of the QPI pattern in the real-space LDOS with high and low voltages, which suggest the surface Dirac electrons alter their wavelength to adjust to the underlying bulk disorder potential, and are not immune to such perturbations. Whereas such fluctuations in momentum are a relatively weak perturbation to the Dirac electrons with high energies; near the Dirac point they are comparable to the average value of the momentum. The lack of well-defined momentum near the Dirac point due to the fluctuations reported is also likely to play a role in the apparent suppression of the ARPES measured signals in magneticallydoped TIs advocated recently ${ }^{36 \mid 37}$

Madhavan's group used the STM and STS to investigate the effects of one-dimensional buckling on the electronic properties of $\mathrm{Bi}_{2} \mathrm{Te}_{3}{ }^{[38}$ By tracking spatial variations of the interference patterns generated by the Dirac electrons, they show that buckling imposes a periodic potential, which locally modulates the SS dispersion. Such potential induces the scattering along $\bar{\Gamma}-\bar{M}$ direction, which support the absence of backscattering and wellnesting of the snowflake-like CEC.

\section{CONCLUSION}

The theoretical and experimental investigations indicate that the LDOS oscillation on the surface of TIs is generally determined by the scattering between surface states around the extremal points on Fermi surface, either by step edges or by non-magnetic impurities. The forbidden backscattering and suppressed standing wave oscillation clearly demonstrate the 2D Dirac nature of topological surface states. The robustness of the topological surface states may have potential application in quantum computing or spintronics in the future.

\section{ACKNOWLEDGMENTS}

This work is supported by the Program of Basic Research Development of China (Grant No. 2011CB921901) and National Natural Science Foundation of China (Grant No. 11074143).
* To whom correspondence should be addressed. bfz@mail.tsinghua.edu.cn

1 M. Z. Hasan and C. L. Kane, Rev. Mod. Phys. 82, 3045 (2010)

${ }^{2}$ X.-L. Qi and S.-C. Zhang, Rev. Mod. Phys. 83, 1057 (2011)

${ }^{3}$ L. Fu and C. L. Kane, Phys. Rev. Lett. 100, 096407 (2008)

${ }^{4}$ X.-L. Qi, R. Li, J. Zang, and S.-C. Zhang, Science 323, $1184(2009)$

${ }^{5}$ R. Li, J. Wang, X. L. Qi, and S. C. Zhang, Nature Phys. 6, 284 (2010).
${ }^{6}$ L. Fu and C. L. Kane, Phys. Rev. B 76, 045302 (2007).

7 D. Hsieh, D. Qian, L. Wray, Y. Xia, Y. S. Hor, R. J. Cava, and M. Z. Hasan, Nature 452, 970 (2008).

${ }^{8}$ D. Hsieh, Y. Xia, L. Wray, D. Qian, A. Pal, J. H. Dil, J. Osterwalder, F. Meier, G. Bihlmayer, C. L. Kane, Y. S. Hor, R. J. Cava, and M. Z. Hasan, Science 323, 919 (2009).

9 Y. Xia, D. Qian, D. Hsieh, L. Wray, A. Pal, H. Lin, A. Bansil, D. Grauer, Y. S. Hor, R. J. Cava, and M. Z. Hasan, Nature Phys. 5, 398 (2009).

10 H. Zhang, C.-X. Liu, X.-L. Qi, X. Dai, Z. Fang, and S.-C. Zhang, Nature Phys. 5, 438 (2009). 
11 D. Hsieh, Y. Xia, D. Qian, L. Wray, J. H. Dil, F. Meier, J. Osterwalder, L. Patthey, J. G. Checkelsky, N. P. Ong, A. V. Fedorov, H. Lin, A. Bansil, D. Grauer, Y. S. Hor, R. J. Cava, and M. Z. Hasan, Nature 460, 1101 (2009).

12 Y. L. Chen, J. G. Analytis, J.-H. Chu, Z. K. Liu, S.-K. Mo, X. L. Qi, H. J. Zhang, D. H. Lu, X. Dai, Z. Fang, S. C. Zhang, I. R. Fisher, Z. Hussain, and Z.-X. Shen, Science 325, 178 (2009)

is L. Petersen, P. T. Sprunger, P. Hofmann, E. Lægsgaard, B. G. Briner, M. Doering, H.-P. Rust, A. M. Bradshaw, F. Besenbacher, and E. W. Plummer, Phys. Rev. B 57, R6858 (1998)

${ }^{14}$ M. F. Crommie, C. P. Lutz, and D. M. Eigler, Nature 363, 524 (1993)

is C. Wittneven, R. Dombrowski, M. Morgenstern, and R. Wiesendanger, Phys. Rev. Lett. 81, 5616 (1998)

16 J. E. Hoffman, K. McElroy, D.-H. Lee, K. M. Lang, H. Eisaki, S. Uchida, and J. C. Davis, Science 297, 1148 (2002)

${ }^{17}$ G. M. Rutter, J. N. Crain, N. P. Guisinger, T. Li, P. N. First, and J. A. Stroscio, Science 317, 219 (2007).

18 P. Roushan, J. Seo, C. V. Parker, Y. S. Hor, D. Hsieh, D. Qian, A. Richardella, M. Z. Hasan, R. J. Cava, and A. Yazdani, Nature 460, 1106 (2009)

19 T. Zhang, P. Cheng, X. Chen, J.-F. Jia, X. Ma, K. He, L. Wang, H. Zhang, X. Dai, Z. Fang, X. Xie, and Q.-K. Xue, Phys. Rev. Lett. 103, 266803 (2009).

20 H. Beidenkopf, P. Roushan, J. Seo, L. Gorman, I. Drozdov, Y. S. Hor, R. J. Cava, and A. Yazdani, Nature Phys. 7, 939 (2011).

${ }^{21}$ X. Zhou, C. Fang, W.-F. Tsai, and J. Hu, Phys. Rev. B 80, $245317(2009)$

${ }^{22}$ W.-C. Lee, C. Wu, D. P. Arovas, and S.-C. Zhang, Phys. Rev. B 80, 245439 (2009)

${ }^{23}$ Q.-H. Wang, D. Wang, and F.-C. Zhang, Phys. Rev. B 81, $035104(2010)$

${ }^{24}$ H.-M. Guo and M. Franz, Phys. Rev. B 81, 041102 (2010).

25 R. R. Biswas and A. V. Balatsky, Phys. Rev. B 83, 075439 (2011)

20 J. Wang, W. Li, P. Cheng, C. Song, T. Zhang, P. Deng, X. Chen, X. Ma, K. He, J.-F. Jia, Q.-K. Xue, and B.-F.
Zhu, Phys. Rev. B 84, 235447 (2011)

27 Z. Alpichshev, J. G. Analytis, J.-H. Chu, I. R. Fisher, Y. L. Chen, Z. X. Shen, A. Fang, and A. Kapitulnik, Phys. Rev. Lett. 104, 016401 (2010)

${ }_{28}$ T. Zhang, N. Levy, J. Ha, Y. Kuk, and J. A. Stroscio, Phys. Rev. B 87, 115410 (2013).

29 Y. Okada, C. Dhital, W. Zhou, E. D. Huemiller, H. Lin, S. Basak, A. Bansil, Y.-B. Huang, H. Ding, Z. Wang, S. D. Wilson, and V. Madhavan, Phys. Rev. Lett. 106, 206805 (2011)

${ }^{30}$ L. Fu, Phys. Rev. Lett. 103, 266801 (2009)

31 L.-T. Huang and B.-F. Zhu, Proceedings of the 31st International Conference on the Physics of SemiconductorsZurichJuly 29-Aug. 4,2012.

${ }^{32}$ K. Kuroda, M. Arita, K. Miyamoto, M. Ye, J. Jiang, A. Kimura, E. E. Krasovskii, E. V. Chulkov, H. Iwasawa, T. Okuda, K. Shimada, Y. Ueda, H. Namatame, and M. Taniguchi, Phys. Rev. Lett. 105, 076802 (2010)

${ }^{33}$ L. M. Roth, H. J. Zeiger, and T. A. Kaplan, Phys. Rev. 149, $519(1966)$

34 J. Seo, P. Roushan, H. Beidenkopf, Y. S. Hor, R. J. Cava, and A. Yazdani, Nature 466, 343 (2010)

35 M. Ye, S. V. Eremeev, K. Kuroda, E. E. Krasovskii, E. V. Chulkov, Y. Takeda, Y. Saitoh, K. Okamoto, S. Y. Zhu, K. Miyamoto, M. Arita, M. Nakatake, T. Okuda, Y. Ueda, K. Shimada, H. Namatame, M. Taniguchi, and A. Kimura, Phys. Rev. B 85, 205317 (2012).

${ }^{36}$ Y. L. Chen, J.-H. Chu, J. G. Analytis, Z. K. Liu, K. Igarashi, H.-H. Kuo, X. L. Qi, S. K. Mo, R. G. Moore, D. H. Lu, M. Hashimoto, T. Sasagawa, S. C. Zhang, I. R. Fisher, Z. Hussain, and Z. X. Shen, Science 329, 659 (2010)

37 L. A. Wray, S.-Y. Xu, Y. Xia, D. Hsieh, A. V. Fedorov, Y. S. Hor, R. J. Cava, A. Bansil, H. Lin, and M. Z. Hasan, Nature Phys. 7, 32 (2011).

38 Y. Okada, W. Zhou, D. Walkup, C. Dhital, S. D. Wilson, and V. Madhavan, Nature Commun. 3, 1158 (2012). 